\title{
The Role of an Implicit Assumption of Causality in the Methodology of Empirical Research
}

\author{
Anna Storozhuk \\ The Institute of Philosophy and Law, Siberian Branch of the Russian Academy of Science, Novosibirsk, Russia \\ Email: stor71@mail.ru
}

How to cite this paper: Storozhuk, A (2018). The Role of an Implicit Assumption of Causality in the Methodology of Empirical Research. Open Journal of Philosophy, $8,308-316$.

https://doi.org/10.4236/ojpp.2018.83022

Received: April 2, 2018

Accepted: May 26, 2018

Published: May 29, 2018

Copyright (C) 2018 by author and Scientific Research Publishing Inc. This work is licensed under the Creative Commons Attribution International License (CC BY 4.0).

http://creativecommons.org/licenses/by/4.0/

\begin{abstract}
The purpose of the article is to point out the causal ladenness of empirical data in the social sciences. This is a kind of theory ladenness, representing implicit assumptions about the deterministic nature of political processes. The nonlinear and chaotic nature of social phenomena requires the collection of data not only about the current state of the system, but also about the evolution of the system. Using an example, we illustrate that the conclusions made on the basis of information about the final state can be very different from the conclusions made on the basis of monitoring the dynamics of the system. Low-importance factors can have big consequences in a chaotic case and, vice versa, there takes place fading of causality: considerable efforts can lead to more than modest results. For the successful management of political life, it is important to be able to identify the impacts that lead to great consequences.
\end{abstract}

\section{Keywords}

Theory-Laden Empirical Data, Determinism, Chaotic Causality

\section{Introduction}

Empirical research in the humanities is based on the collection of data through questionnaires, opinion polls and other methods. These data, used for further analysis, are considered as the starting point, the basis for further conclusions. Such an approach conceals in itself an implicit assumption about the determinacy of certain social processes by their external manifestations. We make this assumption implicitly, assuming causal determinism, that is, a rigid linear connection between causes and effects. According to this assumption, each phenomenon has its own cause and the relation between them is direct and unambiguous: one cause leads, in some definite way, to a single effect. This corresponds to such a feature of human thinking as determinism or the presence of rigid 
cause-effect connections. Strictly speaking, causal determinism, often implicitly assumed by us both in scientific research and in ordinary life, is only valid when there is a major very significant cause that clearly influences occurrence of an event. However, in real life, we often have to deal with a lot of factors of little significance that lead to the result all in the aggregate. In this case, the use of causal determinism is incorrect, and often a chaotic behavior better corresponds to such cases. Below we will consider other approaches to understanding causality that include consideration of chaotic types of causality, such as divergence of solutions, nonlinearity and instability with respect to initial data. A peculiarity of the chaotic understanding of causality is the impossibility of determining a unique connection between the final and the initial state of the system by means of a single-valued relation. In order to make a conclusion about the final state of the system in the case of a chaotic causal connection, step-by-step tracking of the system states in time is required, since at each step the path of development of the system can be changed under the influence of external factors, even if the latter are insignificant.

Below we consider an example where the conclusions drawn from the final analysis of the data may significantly differ from the conclusions made in considering the dynamics of the evolution of the system under consideration.

\section{Are the Empirical Data Pure or Theory-Laden?}

The program of empirical research received a methodological justification in the studies of logical positivism, in particular, by the thinkers of Vienna Circle (Carnap, Hahn, \& Neurath, 1929). It was a community of scientists of various specialties (mathematics and logic, physics, economists, etc.) Briefly, their methodological guideline was the "logic and experience" motto. They perceived science as built with the help of analysis and mathematical processing of initial data. The latter were understood as an independent and objective source of data about the world.

However, later on, the question of how to obtain data turned out to be controversial. Several problem points were identified, one of which was theory-ladenness (for more details, see: Adam, 2004; Franklin et al., 1989). This formidable argument of philosophical theory is similar to the assertion that wherever we lose the keys, we will look for them under the lantern, because it is lighter there. That is, while investigating nature and society, we will pay attention to those phenomena, about which our theory tells us. Not because such data will be the most significant, but simply because this is the first thing that comes to our mind. In addition to the fact that theoretical preferences tell us "where to look", they also participate in the interpretation of what has been seen and treat the collected data in one way or another.

More rigorously speaking, the term "theoretical ladenness of observations" is understood in several senses.

- First of all, it means that the obtained data are described in a language con- 
taining theoretical terms. That is, when measuring the electric current, the physicist says: "The ammeter shows a current of 2 Amps," and not the phrase "The device's arrow has swung to the right." The last phrase could be an example of "pure observation", which does not contain any theoretical interpretation. Theoretical ladenness in this sense was first introduced by P. Duhem in his book "Physical Theory, its Purpose and Structure". Duhem thought that any measurement with the help of any device always introduces theoretical ladenness (Duhem, 1954).

- Theoretical ladenness has begun to be understood in a broader sense starting with the work "Patterns of discovery" by N. Hanson (Hanson, 1958: p. 3). $\mathrm{He}$ believed that our assumptions define the vision itself, and not just its interpretation. Hanson begins the book with an example, when two microbiologists look at the same microscope and, answering the question "what do you see?", one of them mentions the structure of the cell, while the other, the organs of motion, nutrition, etc. The difference in their responses is due to the fact that one considers amoeba to be a cell, while the other, an animal.

- Under the influence of Gestalt psychology, T. Kuhn (1970) expanded the notion of theoretical ladenness even further, understanding the latter, in fact, as a paradigm as a whole. He believed that it is the paradigm that directs research and determines which aspects of reality should be investigated first and which ones are of no significance for illustrating the paradigm.

In this list, each subsequent interpretation can be regarded as an extension of the previous ones, since with the increase of the number, new meanings are added to the original concept.

The "theory-ladenness" term is not necessarily connected with a scientific theory. It can be just contexts that change the shades of meaning, for example, "to fall" is a death in battle and "to decease" is a death from natural causes. Also, one of the types of ladenness of observation is the causal ladenness (Heidelberger, 2003). The question of whether scientific observations are pure or laden is debatable in philosophy. The basic point of view is that although in modern science most of the observations are recognized as laden, but nevertheless, philosophers argue for the presence of at least a small number of pure observations.

What assumptions are often made when collecting empirical data in social sciences?

\section{Three Paradigms of Causality}

The purpose of this discussion will be to identify an implicit assumption that is often made in the course of empirical research.

1) Determinism. A assumption about the causal predetermination of the world is called determinism. Its authorship is attributed to Laplace, who wrote that if all the laws operating in the world and all the initial conditions were known, then it would be possible to definitively calculate the state of affairs in the universe at any time. This is too optimistic. 
The problem is that determinism is an overly strong simplification. Its roots go back to Aristotle, who believed that matter is absolutely passive, accepting external influences. Respectively, the cause of movement always lay outside. It was already the positivist J. S. Mill who wrote that this is too crude an approximation. He said that determinism is a necessary simplification of nature in order to provide the possibility of cognition as such. We accept that every consequence is connected solely to one cause alone, which is not true, since the phenomenon can be produced by different causes. The consequences of various causes may turn out to be not heterogeneous, but homogeneous and not separable from each other by any specific boundary; "the whole of which circumstances perhaps constituted in this particular case the conditions of the phenomenon, or, in other words, the set of antecedents which determined it, and but for which it would not have happened. The real Cause is the whole of these antecedents; and we have, philosophically speaking, no right to give the name of cause to one of them, exclusively of the others" (Mill, 1882: p. 237).

2) A non-linear paradigm of causality presupposes not only a multiplicity of factors, but also their interaction. Unlike determinism, where an object is seen as a passive receiver of external influence, the non-linear paradigm takes into account the internal activity of the objects themselves. So, even in the case of forced mechanical motion, one must reckon with the forces of inertia that counteract external causes (the third Newton's law reflects this circumstance: the action is equal to the counteraction).

The non-linear paradigm of causality presupposes the interaction of external acting forces and the unequal connection between causes and effects. The latter was noticed as early as in 1897 by the Italian economist V. Pareto, who analyzed the incomes of Italian families. He revealed an uneven distribution of finances: the major part of income belongs to a smaller part of families, whereas most families have very modest incomes. Later, the "20/80 principle" was formulated, according to which $20 \%$ of causes have $80 \%$ of consequences and vice versa. This principle was called the Pareto rule. The numerical values, indicated in this principle, are approximate; in some cases it may be a ratio of 30/70 or some other. That is, from the Pareto's point of view, the uneven distribution of income is a consequence of the non-linear nature of causality, leading to an imbalance between the effort expended and the result obtained, and not simply a consequence of the exploitation of workers.

This rule turned out to be universal and concerns not only the economy, but almost any process: for example, we spend only $20 \%$ of time in the most efficient way and this portion brings us $80 \%$ of the results, the rest of time is spent on only $20 \%$ of the work (Koch, 2013).

In essence, nonlinearity leads to the rejection of causality, since the latter presupposes the possibility of opposing the active principle (cause) to the passive one (effect). The axiological consequences lead to significant shifts in the worldview, since the picture of the world in the framework of this approach becomes significantly more complicated and requires the consideration of some 
hidden implicit factors. In the case of economy, this leads to a revision of planning and the search for a winning strategy, based on an analysis that highlights the most effective causes.

Already in the case of non-linear causality, we find ourselves in the situation of impossibility to judge the dynamics of the system based on its finite state. In this case, one cannot rely simply on the collected empirical data, since they characterize only the final state of the system and do not always allow tracking all the stages of its development.

As an example of importance of the influence of the intermediate stages of the system development, we consider the dynamics of putting dislikes to video clips in YouTube (https://www.youtube.com). This platform is arranged so that if a video gets more dislikes than the likes, then it is moved to the end of the list. On Figure 1, the blue curve shows increase of likes in time. It has a smooth shape, which indicates its natural origin: likes were made gradually during seeing video. Other yellow curves show a dislike statistic. It has a step-like nature. The graph shows that the number of dislikes is very quickly increases for a very short time. It's point on then in a very short time it increased sharply, which is clearly artificial, so there are places when in a few seconds the number of dislays increased by several hundred. Thus, we see that many opposition videos have more dislikes than the likes, which allows one to appraise highly the official policy.

Consider, however, a system that allows tracking the dynamics of putting dislikes in time. The graphs of growth of the number of dislikes for the opposition

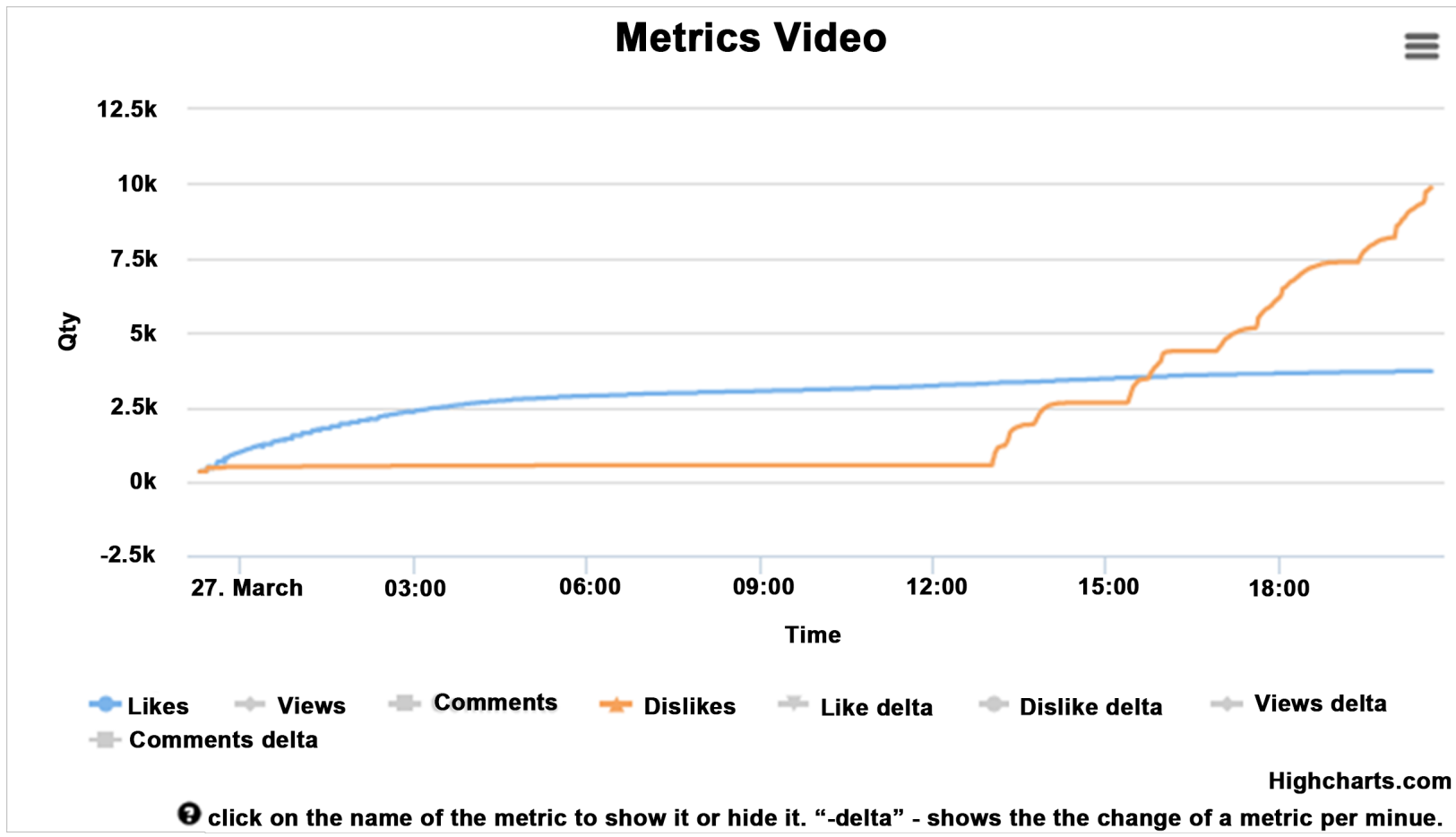

Figure 1. The graph shows that the increase in the number of likes is gradual, whereas the increase of dislikes is jump-like. Moreover, in the region of steps of the graph of dislikes, there are segments where the addition of more than 500 dislikes occurs during 5 seconds. 
videos are step-like, which allows drawing another conclusion, namely, that the number of dislikes is not an expression of public opinion.

\section{Chaotic Causality}

Very often it is impossible to identify the main underlying cause. Phenomena occur during the confluence of a number of random circumstances, as a result of the action of a large number of random factors. It is important to note that, despite the chaotic nature, this situation can be described mathematically, in particular, with the help of a normal distribution. The latter, as the name suggests, very often occurs in nature and characterizes a certain scatter of values. The normal distribution of many quantities makes it possible to understand the quantitative ratio of rare and frequent events. The axiological influence of the chaotic approach to causality is especially noticeable among economists, who in their practice have been convinced of the chaotic nature of the market to a great extent. R. Koch (2001) writes that the major part of business is non-linear and unpredictable, whereas each of the various business areas follows certain, clearly traceable models. There are always several powerful forces that we can use for our own benefit, and those that are capable of destroying our plans. Success usually does not come where we are looking for it, but unexpected successes, if we condescend to notice them, can be easily turned into gold fields. Often additional efforts and capital investments in business result in a decrease in profitability, while the most important economic phenomenon at the start of the 21st century is the growing profitability, when additional investments and intelligent management of intellectual property cause exponential growth in profits.

From the point of view of possibilities of cognizing chaotic behavior and controlling chaos, the mood as a whole is rather optimistic: the principle "if the mess cannot be prevented, it can be headed" is reflected in the methods of the so-called "color" revolutions. "The emergence of new forms and methods of geopolitical rivalry in the Greater Middle East, particularly in the form of the 'theory of controlled chaos', the author of which is an American diplomat and political analyst Steven Mann (Mann, 1997), contributes greatly to scientific, theoretical and conceptual significance of the urgent problems of present-day international relations" (Suleymanova, 2017: p. 190). Thus, the inability to know in detail the immense number of operating factors does not exclude the possibility of isolating the general regularities that govern chaotic behavior. This shows that the development of events is subject to the influence of insignificant and seemingly insignificant factors, which, nevertheless, have significant consequences.

\section{Conclusion}

Empirical research is collecting data that, after mathematical processing, are interpreted. The collected data make it possible to create a general summary picture; it can be static, or it can record an increase or decrease of the observed quantities. Very often, implicitly, the method of collection presupposes a certain 
relationship between external manifestations and hidden processes. Accepting this assumption, the researcher makes judgement about the state of the object under study (for example, society), according to the picture formed from the data analysis.

However, such an approach does not have sufficient reliability, since the relationships between external manifestations (phenomena) and the causal processes that caused them are not always unambiguous. In fact, the area of application of the assumptions about the deterministic type of causality is limited to very few cases when it is possible to identify the main most significant cause which is active, the object of influence is completely passive (does not have internal activity), and the system as a whole is well isolated (to avoid uncontrolled external influences).

In all other cases, the statement that on the basis of the collected data it is possible to obtain an adequate picture of the actual situation is incorrect. Accordingly, this way of thinking in the research (namely, determinism, the assumption of an unambiguous connection between causes and effects) does not allow reliably revealing the reasons for the change in the observed characteristics.

Distortions of the final picture arise due to a number of factors. For example, the active behavior of the object under investigation in response to external influences can change the final picture beyond recognition. The majority of objects and all complex systems (from living organisms to public institutions) manifest their own activity. Further, many small causes interacting with each other can either fade (lose the power of influence), or on the contrary, enhance each other's actions, just as swings are driven to resonance. In such cases, the smallest causes can have colossal consequences and vice versa, considerable efforts can result in a meager result.

A minor influence on the system may play a role when the system is in an unstable state. An example is a ball on a convex surface, when the slightest push is enough to roll it to some particular direction. When the system has the ability to exponentially grow, a strong influence of the initial conditions is manifested: a sufficiently small influence in the beginning suffices to make final states very different from each other. In such cases, it is necessary to take into account the nonlinear and even chaotic nature of causality.

The deterministic mindset as the most early formed continues to dominate public thinking. Even in the cases of chaotic processes, chaos is still considered as a manifestation of limitations of our knowledge. The idea of multiple causality is difficult to grasp; often the most obvious factors of increase/decrease are indicated, but the most significant acting factors may not be revealed in the course of empirical analysis. In this case, the action they produce may be erroneously attributed to other causes.

To obtain a more adequate summary picture based on empirical data, it will be useful to collect information on the intermediate state of the system. The purpose of obtaining intermediate data is to determine the general form of the dynamics. If the relation between the influences on the object and its reactions is 
linear, then it is sufficient to have a deterministic approach. If there is nonlinearity, then it makes sense to obtain data on the degree of stability of the system and its chaoticity. In the latter case, some specific regularities hold, for example, the law of normal distribution, which occurs in the case of several random factors. But the interpretation of such data and recommendations for changing the state of affairs should be different.

Empirical research allows giving a general summary picture, which fixes the growth or loss of the observed quantities. However, it does not allow us to reliably reveal the reasons for the change in the observed characteristics. The deterministic mindset, as the one that was first formed, continues to dominate in social thinking. Even in the cases of chaotic processes, chaos continues to be considered as a manifestation of the limitation of our knowledge. The idea of multiple causality is difficult to grasp; often the most obvious factors of growth/decrease are indicated, but the most significant acting factors may not be revealed in the course of empirical analysis. In this case, the action they produce may be erroneously attributed to other reasons.

\section{References}

Adam, M. (2004). Why Worry about Theory-Dependence? Circularity, Minimal Empiricality and Reliability. International Studies in the Philosophy of Science, 18, 117-132. https://doi.org/10.1080/0269859042000296486

Carnap, R., Hahn, H., \& Neurath, O. (1929). WissenschaftlicheWeltauffassung-Der Wiener Kreis. Wien: Artur Wolf Verlag. http://neurath.umcs.lublin.pl/manifest.pdf

Duhem, P. (1954). The Aim and Structure of Physical Theory. Princeton, NJ: Princeton University Press.

Franklin, A., Anderson, M., Brock, D., Coleman, S., Downing, J., Gruvender, A., Lilly, J., Neal, J., Peterson, D., Price, M., Rice, R., Smith, L., Speirer, S., \& Toering, D. (1989). Can a Theory-Laden Observation Test a Theory? British Journal for the Philosophy of Science, 40, 229-331. https://doi.org/10.1093/bjps/40.2.229

Hanson, N.R. (1958) Patterns of Discovery an Inquiry into the Conceptual Foundations of Science. New York: Cambridge University Press.

Heidelberger, M. (2003).Theory-Ladenness and Scientific Instruments in Experimentation. In H. Radder (Ed.), The Philosophy of Scientific Experimentation (pp. 138-151). Pittsburgh, PA: University of Pittsburgh Press. https://doi.org/10.2307/j.ctt5hjsnf.11

Koch, R. (2001). The Natural Laws of Business: How to Harness the Power of Evolution, Physics, and Economics to Achieve Business Success. New York: Doubleday.

Koch, R. (2013) The 80/20 Manager: The Secret to Working Less and Achieving More. Little, Brown and Company. Hachette Book Group.

Kuhn, T. S. (1970). The Structure of Scientific Revolutions. Enlarged (2nd ed., p. 210). Chicago, IL: University of Chicago Press.

Mann, S. R. (1997). The Reaction to Chaos. In D. S. Alberts, \& T. J. Czerwinski (Eds.), Complexity, Global Politics, and National Security (pp. 62-68). Washington, DC: National Defense University.

Mill, J. S. (1882). A System of Logic, Ratiocinstive and Inductive, Being a Connected View of the Principles of Evidence, and the Methods of Scientific Investigation (8th ed.). 
New York: Harper \& Brothers.

Suleymanova, L. (2017). The Influence of the "Controlled Chaos" Technology on the Geopolitical Situation in the Greater Middle East. Open Journal of Political Science, 7, 189-196. https://doi.org/10.4236/ojps.2017.72015 